\title{
Comments on "Asymptotic Eigenvalue Distributions and Capacity for MIMO Channels Under Correlated Fading” [1]
}

\author{
Georgy Levin and Sergey Loyka, Senior Member, IEEE
}

\begin{abstract}
A stronger and general sufficient condition for the asymptotic normality of MIMO channel eigenvalues and its capacity is given. Physical interpretation of this condition is discussed. Simple alternative conditions, which do not require eigenvalue decomposition, are proposed. It is demonstrated that some popular correlation matrix models satisfy these conditions. In many cases, the convergence to the asymptotic normality is at least as $1 / \sqrt{n_{t}}$, where $n_{t}$ is the number of $\mathbf{T x}$ antennas.
\end{abstract}

Index Terms-Asymptotic analysis, correlated fading, MIMO channel, outage capacity.

W HILE the exact eigenvalue distribution of MIMO channels ${ }^{1}$ is rather complex, Martin and Ottersten [1] proposed a simple and well-tractable asymptotic approximation. In particular, they proved that when the number of antennas at the Tx end is large, the eigenvalues of a correlated Rayleigh fading channel are uncorrelated and asymptotically Gaussian. This result immediately implies that the outage capacity distribution of such a channel is also asymptotically Gaussian, since the capacity is a continuously differentiable function of the eigenvalues [1].

Some comments that extend and strengthen the results in [1] are given below. We adopt the original notations and normalizations in [1], and assume that $\mathbf{Q}=\mathbf{I} \cdot P / n_{t}$.

Comment 1: Generalized Convergence Condition. [[1], Theorem 1] gives a condition under which the eigenvalues of a correlated Rayleigh MIMO channel are uncorrelated and asymptotically Gaussian as $n_{t} \rightarrow \infty$, which follows from the Liapounoff Central Limit Theorem. The generality of this condition can be further extended without any increase in complexity. Specifically, from a more general formulation of Liapounoff Theorem ${ }^{2}$, the generalized condition for [[1], Theorem 1] is that, for some $\delta>0$,

$$
\lim _{n_{t} \rightarrow \infty} Z_{n_{t}}(\delta)=\lim _{n_{t} \rightarrow \infty} \frac{\left\|\lambda^{t}\right\|_{2+\delta}}{\left\|\lambda^{t}\right\|_{2}}=0
$$

where the norm $\left\|\lambda^{t}\right\|_{m}=\left(\sum_{i=1}^{n_{t}}\left(\lambda_{i}^{t}\right)^{m}\right)^{1 / m}$, and $\lambda^{t}=$

Manuscript received September 11, 2006; revised March 26, 2007; accepted June 18, 2007. The associate editor coordinating the review of this letter and approving it for publication was M. Shafi. This work was supported in part by the Natural Sciences and Engineering Research Council of Canada.

The authors are with the School of Information Technology and Engineering, University of Ottawa, Ontario, Canada, K1N 6N5 (e-mail: glevin@site.uottawa.ca: sergey.loyka@ieee.org).

Digital Object Identifier 10.1109/TWC.2008.060699.

${ }^{1}$ We adopt the original terminology and notations in [1]: the eigenvalues of $\mathbf{H H}^{H}$ are termed the channel eigenvalues, where $\mathbf{H}$ is the channel matrix.

${ }^{2}$ Initially, in 1900, Liapounoff showed that a sum of independent random variables $x_{n}$ is asymptotically Gaussian if $E\left(\left|x_{n}\right|^{3}\right)$ exists, and $\sqrt[3]{m_{3}} / \sqrt[2]{m_{2}} \rightarrow 0$ as $n \rightarrow \infty$, where $m_{k}=\sum_{i=1}^{n} E\left(\left|x_{n}\right|^{k}\right)$. Shortly after, in 1901, he found that it is enough to request existence of only some absolute moments $E\left(\left|x_{n}\right|^{2+\delta}\right), \delta>0$, and the sum is asymptotically Gaussian if $2+\delta \sqrt{m_{2+\delta}} / \sqrt[2]{m_{2}} \rightarrow 0$ as $n \rightarrow \infty$ [[2], Ch. 8]. $\left\{\lambda_{i}^{t}, i=1 . . n_{t}\right\}$ is the vector of eigenvalues of the correlation matrix $\mathbf{R}_{t}$. Furthermore, a stronger result holds, as indicated below.

Lemma 1: If $\lim _{n_{t} \rightarrow \infty} Z_{n_{t}}(\delta)=0$ for some $\delta>0$, then it also holds for all $\delta>0$.

Proof: Assume that $\lim _{n_{t} \rightarrow \infty} Z_{n_{t}}\left(\delta_{0}\right)=0$ for some $\delta_{0}>0$. It follows from Liapounoff's Inequality [2], [3] that $\left(\left\|\lambda^{t}\right\|_{2+\delta} /\left\|\lambda^{t}\right\|_{2}\right)^{1 / \delta} \leq\left(\left\|\lambda^{t}\right\|_{2+\delta_{0}} /\left\|\lambda^{t}\right\|_{2}\right)^{1 / \delta_{0}}$ for $\delta \leq \delta_{0}$ and, hence, $Z_{n_{t}}(\delta) \rightarrow 0$ for all $\delta \leq \delta_{0}$. On the other hand, using the norm inequality [[4], Fact 9.7.16], $\left\|\lambda^{t}\right\|_{2+\delta} \leq$ $\left\|\lambda^{t}\right\|_{2+\delta_{0}}$ for $\delta \geq \delta_{0}$ and hence $Z_{n_{t}}(\delta) \rightarrow 0$ for all $\delta \geq \delta_{0}$. Combining the two, Lemma 1 follows.

Based on Lemma 1, we have the following result.

Theorem 1: The eigenvalues of a correlated Rayleigh MIMO channel are uncorrelated and asymptotically Gaussian as $n_{t} \rightarrow \infty$, i.e [[1], Theorem 1] holds, if

$$
\lim _{n_{t} \rightarrow \infty} \frac{\left\|\lambda^{t}\right\|_{\infty}}{\left\|\lambda^{t}\right\|_{2}}=\lim _{n_{t} \rightarrow \infty} \frac{\lambda_{1}^{t}}{\left\|\lambda^{t}\right\|_{2}}=\lim _{n_{t} \rightarrow \infty} \frac{\left\|\mathbf{R}_{t}\right\|_{2}}{\left\|\mathbf{R}_{t}\right\|}=0
$$

where $\lambda_{1}^{t}$ is the maximal eigenvalue of $\mathbf{R}_{t},\left\|\mathbf{R}_{t}\right\|_{2}$ and $\left\|\mathbf{R}_{t}\right\|=$ $\left(\sum_{k, m=1}^{n_{t}}\left|\left[\mathbf{R}_{t}\right]_{k, m}\right|^{2}\right)^{1 / 2}$ are spectral and Frobenius norms respectively [4], $\left[\mathbf{R}_{t}\right]_{k, m}$ are elements of $\mathbf{R}_{t}$.

Proof: Follows immediately from Lemma 1 by choosing $\delta \rightarrow \infty$ in (1).

Due to the inequality $\left\|\lambda^{t}\right\|_{2+\delta} \geq \lambda_{1}^{t}$, the result in Theorem 1 is indeed stronger than those in [[1], Theorem 1] or in (1). Note that evaluation of $\left\|\mathbf{R}_{t}\right\|_{2}$ requires only one (maximal) eigenvalue, Frobenius norm does not require eigenvalue decomposition at all, so that unlike (1), condition (2) is easier to verify. We consider next the convergence rate to the Gaussian distribution assuming that condition (1) holds.

Definition 1: The convergence rate of $Z_{n_{t}}(\delta)$ to zero as $n_{t} \rightarrow \infty$ for given $\delta$ is

$$
R_{Z}(\delta)=\lim _{n_{t} \rightarrow \infty}-\frac{\ln Z_{n_{t}}(\delta)}{\ln n_{t}} \leq \frac{1}{2}-\frac{1}{2+\delta},
$$

where the inequality is due to $Z_{n_{t}}(\delta) \geq n_{t}^{-1 / 2+1 /(2+\delta)}$, which, in turn, follows from Liapounoff's Inequality [[2], Theorem on p. 228].

Proposition 1: The best overall convergence rate is determined by the supremum of (3) taken over all $\delta>0$,

$$
R_{Z}=\sup _{\delta>0} R_{Z}(\delta)=R_{Z}(\infty) \leq 1 / 2,
$$

i.e. in the best possible case $Z_{n_{t}}(\delta) \rightarrow 0$ as $1 / \sqrt{n_{t}}$. A proof follows immediately from the fact that $Z_{n_{t}}(\delta) \geq Z_{n_{t}}(\infty)$ and, consequently, $R_{Z}(\delta) \leq R_{Z}(\infty)$ for any $\delta>0$; the upper bound (the best rate) follows from (3). The best rate is achieved, for example, when all the eigenvalues are equal 
(i.e. no correlation), or when the correlation at the Tx end has a Toeplitz structure (see Comment 4). Note that using a specific fixed $\delta$ to find $R_{Z}$ may lead to an incorrect result ${ }^{3}$, i.e. the supremum in (4) is essential. It should also be pointed out that the generalized Liapounoff Theorem does not require $\delta$ to be a constant [3], [5]: it can be a function of $n_{t}, \delta\left(n_{t}\right)>0$, which further extends the generality of (1). [3] and [5] give specific examples, which demonstrate greater generality of this formulation.

Comment 2: Necessary conditions and some cases when [[1], Theorem 1] does not apply. While the conditions [[1], eq. 10], (1) and (2) are not easy to deal with, some cases when [[1], Theorem 1] does not apply can be characterized in a simple way, which provides simple necessary conditions for that Theorem.

Corollary 1 to [[1], Theorem 1]: Let $\lambda_{1}^{t} \geq \lambda_{2}^{t} \geq \ldots \geq \lambda_{n_{t}}^{t}$ be the ordered eigenvalues of $\mathbf{R}_{t}$. (1) does not hold true, so that [[1], Theorem 1] cannot be applied, if there is a finite set of eigenvalues which are not dominated by the rest, i.e. if there exists $k$ such that

$$
c=\lim _{n_{t} \rightarrow \infty} \frac{\sum_{i=k+1}^{n_{t}}\left(\lambda_{i}^{t}\right)^{2}}{\sum_{i=1}^{k}\left(\lambda_{i}^{t}\right)^{2}}<\infty,
$$

which physically means that the multipath is not rich enough as $n_{t} \rightarrow \infty$.

\section{Proof: see Appendix.}

From (5), a necessary condition for [[1], Theorem 1] is that $c=\infty$. While condition (5) is less general than (1) or (2), it allows for an insight and is simple to evaluate since it involves only the second-order moments of $\lambda^{t}$. Consider two broad cases where Corollary 1 applies: (i) $\mathbf{R}_{t}$ has a finite number ( $k$ ) of non-zero eigenvalues as $n_{t} \rightarrow \infty$, which corresponds to a limited number of multipath in the propagation channel. Then $\sum_{i=k+1}^{n_{t}}\left(\lambda_{i}^{t}\right)^{2}=0$ and consequently $c=0$. Thus, a necessary physical condition for [[1], Theorem 1] to hold is that the number of multipath components goes to infinity with $n_{t}$. (ii) The largest eigenvalue is not dominated by all other eigenvalues, i.e.

$$
c=\lim _{n_{t} \rightarrow \infty} \sum_{i=2}^{n_{t}}\left(\lambda_{i}^{t}\right)^{2} /\left(\lambda_{1}^{t}\right)^{2}<\infty
$$

which hold true, for example, when $\lambda_{2}^{t} \sqrt{n_{t}} / \lambda_{1}^{t}<\infty$ as $n_{t} \rightarrow \infty$. Thus, a necessary condition for [[1], Theorem 1] is that $\lim _{n_{t} \rightarrow \infty} \lambda_{1}^{t} /\left(\lambda_{2}^{t} \sqrt{n_{t}}\right)=0$. Consider, as an example, the uniform correlation matrix [6], when all the non-diagonal entries of $\mathbf{R}_{t}$ are equal to $\rho_{t}$. The eigenvalues in this case can be found explicitly in a closed form: $\lambda_{1}^{t}=1+\left(n_{t}-1\right) \cdot \rho_{t}$, $\lambda_{2}^{t}=\ldots=\lambda_{n_{t}}^{t}=1-\rho_{t}$, where $0 \leq \rho_{t} \leq 1$ is the correlation between two antenna elements. Thus, for $k=1$, $c=0$ if $\rho_{t} \neq 0$, i.e. $\lambda_{1}^{t}$ is not dominated by all the other eigenvalues. In this case it is straightforward to show that, $\lim _{n_{t} \rightarrow \infty} Z_{n_{t}}(\delta)=1$ and [[1], Theorem 1] does not apply.

Comment 3: Upper Bound on the Accuracy of Gaussian Approximation. For finite $n_{t}$, the Gaussian distribution serves as an approximation of the true one. Its accuracy can be estimated from the following results.

\footnotetext{
${ }^{3}$ For example, the results in [1] correspond to $\delta=1$, which implies, without using supremum, $R_{Z} \leq 1 / 6$.
}

Proposition 2: Let $\Delta_{n_{t}}(\mathbf{x})=\left|F_{n_{t}}(\mathbf{x})-\Phi(\mathbf{x})\right|$, where $F_{n_{t}}(\mathbf{x})$ is the CDF of $\lambda$ given $n_{t}$, and $\Phi(\mathbf{x})$ is a Gaussian $\mathrm{CDF}$ with the same mean and covariance as that of $\lambda$. From [[7], Theorem 1.1.] $]^{4}$,

$$
\Delta_{n_{t}}=\sup _{\mathbf{x}} \Delta_{n_{t}}(\mathbf{x}) \leq c \cdot n_{r}^{1 / 4} Z_{n_{t}}(\delta)^{2+\delta}, \quad 0<\delta \leq 1
$$

where $c \leq 4$ is an absolute constant. Moreover, since the channel capacity $J(\lambda)$ (see [1] for the definition) is a continuous function of $\lambda$, and the upper bound in (7) is valid for all $\mathbf{x}$, it also applies to $\Delta_{n_{t}}(x)=\left|F_{n_{t}}(x)-\Phi(x)\right|$, where $F_{n_{t}}(x)$ is the channel outage capacity distribution given $n_{t}$, and $\Phi(x)$ is the Gaussian CDF with the same mean and variance as of $J(\lambda)$.

In analogy with (3), the rate of convergence $\Delta_{n_{t}} \rightarrow 0$ for given $0<\delta \leq 1$ is defined as

$$
R_{\Delta}(\delta)=\lim _{n_{t} \rightarrow \infty}-\frac{\ln \Delta_{n_{t}}}{\ln n_{t}} \geq(2+\delta) R_{Z}(\delta),
$$

where the inequality is due to (3) and (7). From (3), the best convergence rate of $Z_{n_{t}}(\delta) \rightarrow 0$ for given $\delta$ is $1 / 2-1 /(2+\delta)$. In this best case,

$$
R_{\Delta}=\sup _{\delta} R_{\Delta}(\delta) \geq 1 / 2,
$$

i.e. the best convergence is at least as $1 / \sqrt{n}_{t}$. It should be noted that: (i) Even though the lower bound in (9) corresponds to $\delta=1$, it does not necessarily mean that the upper bound in (7) gives the best estimate of $\Delta_{n_{t}}$ when $\delta=1^{5}$. (ii) In some cases, the upper bound in (7) significantly overestimates $\Delta_{n_{t}}$, so that the convergence is better than expected from the bound [5] (see also Comment 7).

Comment 4: Convergence Condition for Toeplitz Matrices. While the conditions in [[1], eq. 10], (1) and (2) are important theoretical tools, their usefulness for practical computations is rather limited due to two reasons: (i) The eigenvalues are known in a closed form only for some simple matrices. Consequently, the aforementioned conditions can be evaluated analytically only in such cases. (ii) Numerical evaluation of these conditions is also difficult, since the numerical complexity (number of operations, inaccuracy, etc.) of the eigenvalue problem increases rapidly with $n_{t}$, so that $n_{t} \rightarrow \infty$ is problematic if possible at all. The following theorem gives a condition that is easier to evaluate.

Theorem 2: Let $\mathbf{R}_{t}$ be a Toeplitz correlation matrix with elements $\left[\mathbf{R}_{t}\right]_{k, m}=t_{k-m}$, such that

$$
0<M_{t}=\lim _{n_{t} \rightarrow \infty} \sum_{k=-n_{t}+1}^{n_{t}-1}\left|t_{k}\right|^{2}<\infty
$$

i.e. $\mathbf{R}_{t}$ is non-degenerate and square-summable ${ }^{6}$. Then for $\forall \delta>0$, the following holds:

$$
\begin{gathered}
\lim _{n_{t} \rightarrow \infty} Z_{n_{t}}(\delta)= \\
=\left(I_{2+\delta}\right)^{1 /(2+\delta)}\left(I_{2}\right)^{-1 / 2} \cdot \lim _{n_{t} \rightarrow \infty} n_{t}^{-\frac{\delta}{2(2+\delta)}}=0
\end{gathered}
$$

${ }^{4}$ [[7], Theorem 1.1.] is stated for $\delta=1$, but it can also be extended to $0<\delta \leq 1[8]$.

${ }^{5}$ See [5] for detailed discussion of this issue.

${ }^{6}$ If $\mathbf{R}_{t}$ is non-degenerate and absolutely summable, it also satisfies (10), since $\sum_{k=-n_{t}+1}^{n_{t}-1}\left|t_{k}\right|^{2} \leq\left(\sum_{k=-n_{t}+1}^{n_{t}-1}\left|t_{k}\right|\right)^{2}$. 
where for $\forall p>0$

$$
I_{p}=(2 \pi)^{-1} \int_{0}^{2 \pi} f^{p}(x) d x<\infty
$$

and a non-negative real function $f(x)=\sum_{k=-\infty}^{\infty} t_{k} \cdot e^{j k x}$ is the spectrum of $\mathbf{R}_{t}$ [9].

Proof: see Appendix.

Not only does Theorem 2 give a practical way to evaluate the condition (1) for Toeplitz correlation matrices ${ }^{7}$ without using eigenvalue decomposition, it also shows that under condition (10), the channel eigenvalues and so the outage capacity are always asymptotically Gaussian ${ }^{8}$. Moreover, it is straightforward to show using Szego Theorem [9], that

$$
M_{t}=\lim _{n_{t} \rightarrow \infty} \sum_{k=-n_{t}+1}^{n_{t}-1}\left|t_{k}\right|^{2}=\lim _{n_{t} \rightarrow \infty} n_{t}^{-1}\left\|\mathbf{R}_{t}\right\|^{2},
$$

where $n_{t}^{-1}\left\|\mathbf{R}_{t}\right\|$ is a measure of correlation and power imbalance of a MIMO channel introduced in [10]. This measure affects the outage capacity distribution and was motivated by asymptotic analysis of the latter, which creates certain analogy with $Z_{n_{t}}(\delta)$. Thus, in the case of Toeplitz matrices, a necessary condition for $Z_{n_{t}}(\delta)$ to converge to zero and hence for [[1], Theorem 1] to hold is that the measure of correlation $n_{t}^{-1}\left\|\mathbf{R}_{t}\right\| \rightarrow 0$ as $n_{t} \rightarrow \infty^{9}$.

Furthermore, from (11), $R_{Z}=1 / 2$, (the supremum is at $\delta \rightarrow \infty$, i.e. under the conditions of Theorem 2, the upper bound in (4) is achieved and the convergence is as $1 / \sqrt{n}_{t}$. This result is general for a wide class of Toeplitz correlation matrices that satisfy (10), regardless of any other details. As a numerical example, Fig. 1 shows the upper bound in (7) and $\Delta_{n_{t}}\left(x_{0}\right)$ vs. $n_{t}$, where $x_{0}$ is the outage capacity such that the outage probability $\Phi\left(x_{0}\right)=0.01 . Z(\delta)$ is calculated at $\delta=1$ for $\mathbf{R}_{t}$ given by the exponential correlation model [11] with correlation parameter $\rho_{t}=0.5, \Delta_{n_{t}}\left(x_{0}\right)$ is obtained by Monte-Carlo (MC) simulation using $10^{5}$ trials. As expected, the upper bound (solid line) decreases as $1 / \sqrt{n}_{t}$ (see the dashed line for comparison). $\Delta_{n_{t}}\left(x_{0}\right)$ lies well below the upper bound, and decreases with $n_{t}$ at least as $1 / \sqrt{n}_{t}$.

Comment 5: Convergence Condition for Arbitrary Matrices. When $\mathbf{R}_{t}$ is not Toeplitz, Theorem 2 does not apply. However, it is shown in the Appendix for correlation matrices with an arbitrary structure, that $Z_{n_{t}}(1)$ is bounded by the norm of $\mathbf{R}_{t}$ as follows:

$$
\left(n_{t}^{-1}\left\|\mathbf{R}_{t}\right\|\right)^{1 / 3} \leq Z_{n_{t}}(1) \leq 1
$$

Similarly to the Toeplitz correlation structures considered above, a necessary condition for $Z_{n_{t}}(1)$ to converge to zero and hence for [[1], Theorem 1] to hold is that the measure of correlation $n_{t}^{-1}\left\|\mathbf{R}_{t}\right\| \rightarrow 0$ as $n_{t} \rightarrow \infty$. Moreover, from [10], $n_{t}^{-1 / 2} \leq n_{t}^{-1}\left\|\mathbf{R}_{t}\right\| \leq 1$, where the lower bound corresponds to the case when $\lambda_{i}^{t}=1, \forall i$, i.e. there is no correlation at the Tx end, and the upper bound is achieved when there is a single non-zero eigenvalue $\lambda_{1}^{t}=n_{t}$ and $\lambda_{i}^{t}=0$ for $\forall i \neq 1$, i.e. the Tx end is fully correlated. Thus, the overall tendency for

\footnotetext{
${ }^{7}$ Toeplitz correlation matrix physically corresponds to a uniform antenna array geometry, when correlation depends on the spacing between elements only, but not on their positions.

${ }^{8}$ Note that the uniform correlation matrix [6] does not satisfy (10), unless $\rho_{t}=0$

${ }^{9}$ In this sense, the Tx antennas have to be "asymptotically uncorrelated".
}

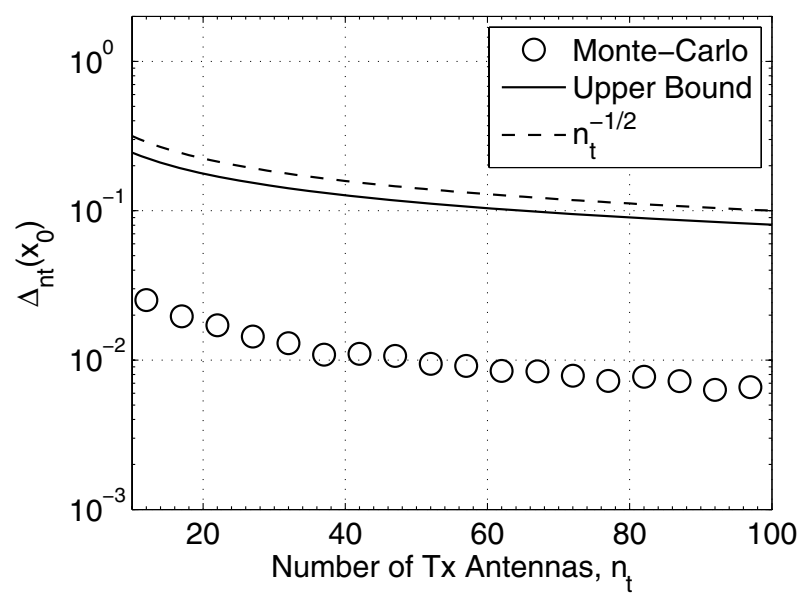

Fig. 1. Distance between the outage capacity distribution of a Rayleigh fading channel and the Gaussian approximation; $c=0.4, n_{r}=2, \Phi\left(x_{0}\right)=0.01$.

$Z_{n_{t}}(1)$ is to increase with correlation, which results in slower convergence for higher correlated channels (see also [10]).

Comment 6: Convergence for Some Popular Correlation Matrices. While the exponential correlation matrix has been used in [1], it was not demonstrated that it satisfies the condition of [[1], Theorem 1]. In fact, the eigenvalues in this case are given by a transcendental equation [12], which does not allow easy evaluation of (1). However, using d'Alambert Ratio Test, it can be shown that in this case $M_{t}<\infty$ for $\left|\rho_{t}\right|<1$, where $\rho_{t}$ is the correlation between adjacent Tx antennas, so that following Theorem 2 in Comment 4, condition (1) is indeed satisfied. Moreover, using (11), for $\left|\rho_{t}\right|<1$

$$
\begin{aligned}
\lim _{n_{t} \rightarrow \infty} Z_{n_{t}}(1) & =\frac{\left(1+4\left|\rho_{t}\right|^{2}+\left|\rho_{t}\right|^{4}\right)^{1 / 3}}{\left(1+\left|\rho_{t}\right|^{2}\right)^{1 / 2} \cdot\left(1-\left|\rho_{t}\right|^{2}\right)^{1 / 6}} \times \\
\times & \lim _{n_{t} \rightarrow \infty} n_{t}^{-1 / 6}=0
\end{aligned}
$$

From the definition of a limit, for any $\epsilon>0$, there is $n_{0}$ such that for all $n_{t}>n_{0}, Z_{n_{t}} \leq \epsilon$. (15) shows that $n_{0}$ is an increasing function of $\left|\rho_{t}\right|$, i.e. larger correlation results in slower convergence. This supports the conclusion in Comment 4, and explains the corresponding observation in [1], which was based on numerical results. When the correlation is significant only among adjacent antennas and can be neglected for other pairs of antennas, the elements of $\mathbf{R}_{t}$ are given by the tri-diagonal correlation matrix [12] for $\left|\rho_{t}\right|<\frac{1}{2}\left(\cos \frac{\pi}{n_{t}+1}\right)^{-1}$,

$$
\left[\mathbf{R}_{t}\right]_{k, m}=\left\{\begin{array}{c}
1, k=m \\
\rho_{t}, k=m-1 \\
\bar{\rho}_{t}, k=m+1 \\
0, \text { otherwise }
\end{array}\right.
$$

where $\bar{\rho}_{t}$ is the complex conjugate of $\rho_{t}$. It is straightforward to show that $M_{t}<\infty$, i.e. from Theorem $2, Z_{n_{t}}(1) \rightarrow 0$ as $n_{t} \rightarrow \infty$. Thus, [[1], Theorem 1] applies in this case. 
Moreover,

$$
\lim _{n_{t} \rightarrow \infty} Z_{n_{t}}(1)=\frac{\left(1+6\left|\rho_{t}\right|^{2}\right)^{1 / 3}}{\left(1+2\left|\rho_{t}\right|^{2}\right)^{1 / 2}} \cdot \lim _{n_{t} \rightarrow \infty} n_{t}^{-1 / 6}=0,
$$

i.e. similarly to (15), $n_{0}$ is an increasing function of $\left|\rho_{t}\right|$ and higher correlation results in slower convergence. Another important case is the squared-exponential correlation matrix defined as

$$
\left[\mathbf{R}_{t}\right]_{k, m}=\left\{\begin{array}{c}
\rho_{t}^{(m-k)^{2}}, k \leq m \\
\bar{\rho}_{t}^{(k-m)^{2}}, k>m
\end{array},\left|\rho_{t}\right| \leq 1\right.
$$

This correlation structure has been proposed for the IEEE 802.11n Wireless LANs standard [13] and describes physical propagation channels where the angular PDF is truncated Gaussian [14], [15]. While the confirmation of (1) is difficult in this case, it is straightforward to show that $M_{t}<\infty,\left|\rho_{t}\right|<$ 1 , and hence from Theorem 2 and [[1], Theorem 1], the channel eigenvalues and so the outage capacity are asymptotically Gaussian.

It can be shown, however, that $M_{t}$ is unbounded for the popular correlation models which correspond to the uniform or truncated Laplacian angular distributions of the multipath. Hence, Theorem 2 does not apply in these cases. Clearly, whether $M_{t}$ is finite or not is determined by asymptotic behavior of $\mathbf{R}_{t}$ 's tails $(k, m \rightarrow \infty)$. However, the match between the popular correlation models and real correlation structures for $k, m \rightarrow \infty$ has not been thoroughly studied, if studied at all, since: (i) in practice $n_{t}$ is always finite, so that the asymptotic behavior of $\mathbf{R}_{t}$ 's tails had little or no importance, and (ii) measuring these tails is difficult from the technical point of view. Thus, the issue of convergence for practical correlation structures seems to be an open problem. The usefulness of Theorem 2, however, is somewhat more general than just with respect to some particular correlation models. From (10), [[1], Theorem 1] applies for all Toeplitz correlation structures for which the correlation decays faster than $1 / \sqrt{D}$, where $D$ is the distance between the antenna elements.

Comment 7: On Practical Utility of Gaussian Approximation. The practical utility of the asymptotic Gaussian distribution is that it can be used as an approximation to the outage capacity distribution of MIMO channels with finite (realistic) $n_{t}$. While the convergence conditions discussed above are important theoretical tools that provide generic guidance, they should be used with caution for practical applications due to the following reasons: (i) Even though condition (1) is satisfied, it does not mean that $\Delta_{n_{t}}$ is sufficiently small for realistic $n_{t}$. Consequently, using Gaussian approximation for realistic (finite) $n_{t}$ may result in inaccurate estimation of the channel capacity. (ii) In the opposite case, when (1) is not satisfied, $\Delta_{n_{t}}$ may be still sufficiently small for given realistic $n_{t}$, so that the Gaussian approximation can be used. Note also that (1) is a sufficient but not necessary condition. (iii) The common generic approach to evaluate $\Delta_{n_{t}}$ theoretically is by the upper bound in (7), which, in many cases, is very conservative for low to moderate $n_{t}$ [5]. As we show above, this bound does not converge faster then $1 / \sqrt{n}_{t}$, which is comparatively slow and requires large $n_{t}$ to guarantee accurate approximation based on the bound alone. In practice, however, the convergence can be much faster, so that the difference between the true distribution and its Gaussian approximation can be indistinguishably small already for $n_{t}=2$, as shown in [16], [17], and in [18] using rigorous statistical methods. This problem arises from the fact that the upper bound in (7) applies to a wide class of channel distributions and therefore cannot be further improved unless specific distributions are considered [19]. The mathematical results in this area are rare $[5]$.

\section{APPENDIX}

Proof of Corollary 1: Consider a lower bound on $Z_{n_{t}}(\delta)$ :

$$
Z_{n_{t}}(\delta)=\frac{\left\|\lambda^{t}\right\|_{2+\delta}}{\left\|\lambda^{t}\right\|_{2}}=\frac{\|\mu\|_{2+\delta}}{\|\mu\|_{2}} \geq\|\mu\|_{2}^{-1}
$$

where $\mu=\left\{\lambda_{i}^{t} / \lambda_{1}^{t}, i=1 . . n_{t}\right\}$. Assume that there is a finite set of $k$ largest eigenvalues which is not dominated by the rest as $n_{t} \rightarrow \infty$, i.e. if $S_{1}=\sum_{i=1}^{k}\left(\mu_{i}\right)^{2}$ and $S_{2}=\sum_{i=k+1}^{n_{t}}\left(\mu_{i}\right)^{2}$, then

$$
c \triangleq \lim _{n_{t} \rightarrow \infty} \frac{S_{2}}{S_{1}}=\lim _{n_{t} \rightarrow \infty} \frac{\sum_{i=k+1}^{n_{t}}\left(\lambda_{i}^{t}\right)^{2}}{\sum_{i=1}^{k}\left(\lambda_{i}^{t}\right)^{2}}<\infty
$$

From (19),

$$
\begin{gathered}
\lim _{n_{t} \rightarrow \infty} Z_{n_{t}}(\delta) \geq \lim _{n_{t} \rightarrow \infty}\left(S_{1}\left(1+S_{2} / S_{1}\right)^{-1 / 2} \geq\right. \\
\geq(k \cdot[1+c])^{-1 / 2}>0
\end{gathered}
$$

where the second inequality is since $S_{1} \leq k$.

Proof of Theorem 2: Since $M_{t}$ is finite, from Szego Theorem [9], the following holds true for $\forall p>0$

$$
\lim _{n_{t} \rightarrow \infty} n_{t}^{-1}\left\|\lambda^{t}\right\|_{p}^{p}=(2 \pi)^{-1} \int_{0}^{2 \pi} f^{p}(x) d x=I_{p}<\infty,
$$

where $f(x)=\sum_{k=-\infty}^{\infty} t_{k} \cdot e^{j k x}$ is a spectrum of $\mathbf{R}_{t}$. Note that since $\mathbf{R}_{t}$ is a correlation matrix, $f(x)$ is non-negative and real. By substituting (22) in (1), one obtains

$$
\lim _{n_{t} \rightarrow \infty} Z_{n_{t}}(\delta)=\left(I_{2+\delta}\right)^{1 /(2+\delta)}\left(I_{2}\right)^{-1 / 2} \cdot \lim _{n_{t} \rightarrow \infty} n_{t}^{\frac{-\delta}{2(2+\delta)}}
$$

Note that both $I_{2}$ and $I_{2+\delta}$ are finite (see (22)) and positive, since $I_{2}=M_{t}>0$ due to Parseval's Theorem, and $\left(I_{2+\delta}\right)^{1 /(2+\delta)} \geq\left(I_{2}\right)^{1 / 2}>0$ due to Liapounoff's Inequality [[2], Theorem p. 228]. Using (23), for $\forall \delta>0$,

$$
\lim _{n_{t} \rightarrow \infty} Z_{n_{t}}(\delta)=0
$$

Proof of (14): Below we adopt the normalization $\operatorname{tr}\left(\mathbf{R}_{t}\right)=$ $n_{t}$ [1]. Lower Bound: First, note that $\left\|\lambda^{t}\right\|_{3}^{3} \geq n_{t}^{-1}\left\|\mathbf{R}_{t}\right\|^{4}$ :

$$
\begin{gathered}
\left\|\lambda^{t}\right\|_{3}^{3}=n_{t}^{-1} \sum_{i=1}^{n_{t}}\left(\left(\lambda_{i}^{t}\right)^{1 / 2}\right)^{2} \cdot \sum_{i=1}^{n_{t}}\left(\left(\lambda_{i}^{t}\right)^{3 / 2}\right)^{2} \geq \\
\geq n_{t}^{-1}\left(\sum_{i=1}^{n_{t}}\left(\lambda_{i}^{t}\right)^{2}\right)^{2}=n_{t}^{-1}\left\|\mathbf{R}_{t}\right\|^{4}
\end{gathered}
$$

where the inequality is due to Cauchy-Schwarz inequality. Thus,

$$
\frac{\left\|\lambda^{t}\right\|_{3}}{\left\|\lambda^{t}\right\|_{2}} \geq \frac{\left(n_{t}^{-1}\left\|\mathbf{R}_{t}\right\|^{4}\right)^{1 / 3}}{\left(\left\|\mathbf{R}_{t}\right\|^{2}\right)^{1 / 2}}=\left(n_{t}^{-1}\left\|\mathbf{R}_{t}\right\|\right)^{1 / 3}
$$


Upper Bound: First, note that $\left\|\lambda^{t}\right\|_{3}^{3} \leq\left\|\mathbf{R}_{t}\right\|^{3}$ :

$$
\begin{gathered}
\left\|\lambda^{t}\right\|_{3}^{3}=\sum_{i=1}^{n_{t}}\left(\lambda_{i}^{t}\right)^{2} \cdot \lambda_{i}^{t} \leq \\
\leq\left(\sum_{i=1}^{n_{t}}\left(\lambda_{i}^{t}\right)^{4}\right)^{1 / 2}\left(\sum_{i=1}^{n_{t}}\left(\lambda_{i}^{t}\right)^{2}\right)^{1 / 2} \leq \\
\leq \sum_{i=1}^{n_{t}}\left(\lambda_{i}^{t}\right)^{2} \cdot\left(\sum_{i=1}^{n_{t}}\left(\lambda_{i}^{t}\right)^{2}\right)^{1 / 2}=\left\|\mathbf{R}_{t}\right\|^{3}
\end{gathered}
$$

where the first inequality is due to Cauchy-Schwarz inequality, and the second one follows from $\left(\sum_{i=1}^{n_{t}}\left(\lambda_{i}^{t}\right)^{4}\right)^{1 / 2} \leq$ $\sum_{i=1}^{n_{t}}\left(\lambda_{i}^{t}\right)^{2}$. Thus,

$$
\frac{\left\|\lambda^{t}\right\|_{3}}{\left\|\lambda^{t}\right\|_{2}} \leq \frac{\left\|\mathbf{R}_{t}\right\|}{\left\|\mathbf{R}_{t}\right\|}=1
$$

\section{ACKNOWLEDGMENT}

The authors would like to thank Prof. Vidmantas K. Bentkus for his valuable comments on Central Limit Theorems.

\section{REFERENCES}

[1] C. Martin and B. Ottersten, "Asymptotic eigenvalue distributions and capacity for MIMO channels under correlated fading," IEEE Trans. Wireless Commun, vol. 3, no. 4, pp. 1350-1358, July 2004.

[2] B. V. Gnedenko, The Theory of Probability. Moscow: Nauka, 1988.

[3] A. M. Liapounoff, "A new form of the theorem on probability limit," in Proc. Russian Imperial Academy of Sciences, Series VIII, 1901, vol. 12, no. 5, pp. 1-24. (Also appears in A.M. Liapounoff, Collected Works, The USSR Academy of Sciences, 1954, vol. 1, pp. 157-176.)

[4] D. S. Bernstein, Matrix Mathematics: Theory, Facts, and Formulas with Applications to Linear Systems Theory. Princeton, NJ: Princeton University Press, 2005.

[5] V. M. Zolotarev, "On real improvements of central limit theorems," in Proc. Mathematical Institute, USSR Academy Sciences, vol. 182, pp. 24-47, 1988.
[6] S. Loyka and J. Mosig, "Channel capacity of $N$-antenna BLAST architecture," IEEE Electron. Lett., vol. 36, no.7, pp. 660-661, Mar. 2000.

[7] V. Bentkus, "A Lyapunov-type bound in $\mathbf{R}^{d}$," Theory Probab. Appl., vol. 49, no. 2, pp. 311-323, 2005.

[8] V. Bentkus, Private Communication, 2007.

[9] R. M. Gray, "On the asymptotic eigenvalue distribution of Toeplitz matrices," IEEE Trans. Inform. Theory, vol. 18, no. 6, pp. 725-730, Nov. 1972.

[10] G. Levin and S. Loyka, "Multi-keyhole MIMO channels: Asymptotic analysis of outage capacity," in IEEE International Symposium Inform. Theory, July 2006, pp. 1305-1309.

[11] G. L. Turin, "On optimal diversity reception, II," IRE Trans. Commun. Syst., pp. 22-31, Mar. 1962.

[12] J. N. Pierce and S. Stein, "Multiple diversity with nonindependent fading," Proc. IRE, vol. 48, pp. 89-104, Jan. 1960.

[13] V. Erceg, et al., "TGn channel models," Proposal for IEEE P802.11 Wireless LANs, Doc. IEEE 802.11-03/940r4, May 2004.

[14] F. Adachi, et al., "Crosscorrelation between the envelopes of $900 \mathrm{MHz}$ signals received at a mobile radio base station site," IEE Proceedings, vol. 133 , no. 6, pp. 506-512, Oct. 1986.

[15] T. S. Chu and L. J. Greenstein, "A semi-empirical representation of antenna diversity gain at cellular and PCS base stations," IEEE Trans. Commun., vol. 45, no. 6, pp. 644-646, June 1997.

[16] P. J. Smith and M. Shafi, "On a Gaussian approximation to the capacity of wireless MIMO systems," in Proc. IEEE International Conf. Commun., Apr--May 2002, vol. 1, pp. 406-410.

[17] B. M. Hochwald, T. L. Marzetta, and V. Tarokh, "Multiple-antenna channel hardening and its implications for rate feedback and scheduling," IEEE Trans. Inform. Theory, vol. 50, no. 9, Sep. 2004.

[18] G. Levin and S. Loyka, "Statistical approach to MIMO capacity analysis in a fading channel," IEEE Veh. Technol. Conf., VTC2004-Fall, Sep. 2004, vol. 3, pp. 1548-1552.

[19] V. V. Petrov, Limit Theorems of Probability Theory: Sequences of Independent Random Variables, New York: Oxford University Press, 1996. 